\title{
ENHANCEMENT OF ELECTRON SATURATED DRIFT VELOCITY IN A QUANTUM WELL BY CONFINEMENT OF POLAR OPTICAL PHONONS
}

\author{
J. Požela, K. Požela, and V. Jucienè \\ Semiconductor Physics Institute, A. Goštauto 11, LT-01108 Vilnius, Lithuania \\ E-mail: pozela@pfi.lt
}

Received 23 November 2005

\begin{abstract}
It is shown that the confined polar optical phonon momentum quantization allows for a strong increase of the electron saturated drift velocity in a narrow quantum well.
\end{abstract}

Keywords: electron drift velocity, quantum wells, PO phonon confinement

PACS: $72.20 . \mathrm{Ht}$, 73.63.Hs, 72.20.Dp

\section{Introduction}

The cutoff frequency $f_{T}$ and maximal gain $g_{\mathrm{m}}$ of a field-effect transistor (FET) are determined by the electron drift velocity $v_{\mathrm{dr}}$ in the device active region $l_{\mathrm{dr}}$ :

$$
f_{T}=\frac{v_{\mathrm{dr}}}{2 \pi l_{\mathrm{dr}}} \quad \text { and } \quad g_{\mathrm{m}} \approx C v_{\mathrm{dr}},
$$

where $C$ is the gate specific capacitance. Modern FETs with micron gates $l_{\mathrm{dr}}$ have $f_{T} \approx(2-3) \cdot 10^{10} \mathrm{~Hz}$ and $g_{\mathrm{m}} \approx 10^{3} \mathrm{mS} / \mathrm{mm}$. The drift velocity at saturation limits these parameters. In main semiconductors, $v_{\text {sat }}=2 \cdot 10^{7} \mathrm{~cm} / \mathrm{s}$. Because of the drift velocity saturation, an increase of the transistor operational speed is achieved by decreasing the gate length $l_{\mathrm{dr}}$ down to submicron and lower dimensions.

In this paper, we consider an alternative way to enhance the transistor speed and gain by means of a drift velocity increase over the saturated value. It has been shown in [1-3] that electron and polar optical phonon confinement in the GaAs quantum well (QW) decreases the inelastic electron-phonon scattering rate and enhances the low-field electron mobility. It has been observed experimentally that the insertion of thin InAs barriers into GaAs QW allows one to increase the electron maximal drift velocity by several times in InAs / GaAs / InAs QW in a high electric field [4]. In this paper, we explain the effect of the great increase of the drift velocity in a narrow QW by quantization of the confined polar optical (PO) phonon momentum component perpendicular to the QW plane.

\section{Drift velocity saturation}

Inelastic electron-PO phonon scattering is the dominant scattering mechanism limiting the increase of the electron drift velocity in a high electric field.

In a stationary case, the balance between the energy acquired from the field and the energy transferred to the emitted phonon allows one to estimate the value of the electron saturated drift velocity as

$$
v_{\mathrm{sat}}^{\mathrm{opt}}=\sqrt{\frac{E_{\mathrm{opt}}}{m}},
$$

where $m$ is the electron effective mass, and $E_{\text {opt }}$ is the optical phonon energy.

The value of $v_{\mathrm{sat}}^{\mathrm{opt}}$ is determined by the material parameters $E_{\text {opt }}$ and effective mass $m$, and does not depend on the electric field. Most of the common semiconductors ( $\mathrm{Ge}, \mathrm{Si}, \mathrm{GaAs}, \mathrm{InP}, \mathrm{GaN}$, etc.) have the optical phonon energy of about $\hbar \omega_{0} \approx 40 \mathrm{meV}$ and the effective mass $m \approx 0.1 m_{0}$. Substitution of these values into Eq. (1) gives the value of the saturated velocity $v_{\mathrm{sat}}^{\mathrm{opt}} \approx 2 \cdot 10^{7} \mathrm{~cm} / \mathrm{s}$ (see, for example, Refs. [5, 6]).

Assuming that the electron drift velocity is inversely proportional to the electron-PO phonon scattering rate, we suppose that the saturated drift velocity

$$
v_{\text {sat }} \sim W_{\hbar \omega_{0}}^{-1},
$$

where $W_{\hbar \omega_{0}}$ is the scattering rate of an electron with the kinetic energy $E_{c} \geq \hbar \omega_{0}$ by a PO phonon. In common semiconductors, $W_{\hbar \omega_{0}}$ is so large that when 
an electron just achieves the optical phonon energy, it emits a PO phonon and loses its drift velocity obtained in an electric field. In that case, Eq. (1) takes place: i. e. $v_{\mathrm{sat}}=v_{\mathrm{sat}}^{\mathrm{opt}}$. At lower $W_{\hbar \omega_{0}}$, the electron can be accelerated in the electric field up to the velocity higher than $v_{\text {sat }}^{\text {opt }}$, before it emits the PO phonon. We identified the $\mathrm{PO}$ phonon emission with the saturation of the electron drift velocity. In Eq. (2) we propose that the increase of the saturated drift velocity is proportional to the decrease of $W_{\hbar \omega_{0}}$.

The increase of the drift velocity over $v_{\text {sat }}^{\text {opt }}$ due to the decrease of $W_{\hbar \omega_{0}}$ is observed in some specific cases involving electron-phonon interaction in short space and time intervals (the overshoot effect) [7-9].

We will consider possibilities to decrease $W_{\hbar \omega_{0}}$ by the PO phonon confinement in a QW.

\section{Electron-phonon scattering rate and maximal drift velocity in a $\mathbf{Q W}$}

Let us compare the scattering rate of electrons confined in a QW by confined PO phonons with that for bulk PO phonons.

The scattering rate of an electron from an initial state with the wave vector $\mathbf{k}_{i}$ and energy $E_{i}$ to a final state with the wave vector $\mathbf{k}_{f}$ and energy $E_{f}$ by emission (absorption) of a PO phonon with the energy $\hbar \omega_{0}$ and wave vector $\mathbf{q}$ is described by

$W_{\mathbf{k}_{\mathbf{i}} \mathbf{k}_{f}}=\frac{2 \pi}{\hbar}\left|M_{i f}\right|^{2}\left(N_{q}+\frac{1}{2} \pm \frac{1}{2}\right) \delta\left(E_{f} \pm E_{i}+\hbar \omega_{0}\right)$,

where

$$
N_{q}=\left[\exp \left(\frac{\hbar \omega_{0}}{k_{\mathrm{B}} T}\right)-1\right]^{-1}
$$

$k_{\mathrm{B}}$ is the Boltzmann constant, and $T$ is the temperature.

In bulk material, the matrix element for isotropic scattering by longitudinal PO phonons [10]

$$
\begin{aligned}
\left|M_{i f}\right|_{\text {bulk }}^{2} & =\delta_{\mathbf{k}_{f}, \mathbf{k}_{i} \pm \mathbf{q}} \frac{1}{L^{3}} \frac{B_{0}}{q^{2}}, \\
B_{0} & =2 \pi e^{2}\left(\frac{1}{\varepsilon_{\infty}}-\frac{1}{\varepsilon_{\mathrm{S}}}\right) \hbar \omega_{0},
\end{aligned}
$$

where $\delta_{\mathbf{k}_{f}, \mathbf{k}_{i} \pm \mathbf{q}}$ is the Kronecker delta, $q$ is the PO phonon wave vector, and $\varepsilon_{\infty}$ and $\varepsilon_{\mathrm{S}}$ are the highfrequency and static dielectric constants, respectively.

The total scattering rate of an electron in the initial state $\left(\mathbf{k}_{i}, E_{i}\right)$ is equal to

$$
W_{\mathbf{k}_{\mathbf{i}}}=\frac{L^{3}}{(2 \pi)^{3}} \int W_{\mathbf{k}_{\mathbf{i}} \mathbf{k}_{f}} \mathrm{~d}^{3} k_{f} .
$$

For a bulk semiconductor case, integration of Eq. (5) gives

$$
W_{k_{i}, \text { bulk }}=W_{0}^{ \pm} \frac{1}{k_{i}} \ln \frac{k_{i}+\sqrt{k_{f 0}^{2}}}{k_{i}-\sqrt{k_{f 0}^{2}}},
$$

where

$$
\begin{gathered}
-k_{f 0}^{2}= \pm k_{\mathrm{opt}}^{2}-k_{i}^{2}, \\
k_{\mathrm{opt}}^{2}=\frac{2 m\left(\hbar \omega_{0}\right)}{\hbar^{2}},
\end{gathered}
$$

and

$$
W_{0}^{ \pm}=\frac{m e^{2}\left(\hbar \omega_{0}\right)}{2 \pi \hbar^{3}}\left(\frac{1}{\varepsilon_{\infty}}-\frac{1}{\varepsilon_{\mathrm{S}}}\right)\left(N_{q}+\frac{1}{2} \pm \frac{1}{2}\right)
$$

is the Fröhlich coupling coefficient of the interaction of electrons with polar optical phonons. Sign "+" (“-”) is for phonon emission (absorbtion) case. Note that according to Eq. (7), interaction with phonon emission is possible when the electron energy is greater than the optical phonon energy.

Electron and phonon momenta quantization in a 2D structure change radically the electron-phonon interaction. For simplicity, we assume that electrons and PO phonons are confined in a common QW of the width $L$.

The scattering rate of a confined electron by confined phonon is equal to

$$
\begin{aligned}
W_{\mathbf{k}_{\mathbf{i}} \mathbf{k}_{f}}(\mathrm{QW})= & \frac{2 \pi}{\hbar}\left|M_{i f}\right|_{\mathrm{QW}}^{2}\left(N_{q}+\frac{1}{2} \pm \frac{1}{2}\right) \\
& \times \delta\left(E_{f} \pm E_{i}+\hbar \omega_{0}^{*}\right), \\
\left|M_{i f}\right|_{\mathrm{QW}}^{2}= & \delta_{\mathbf{k}_{\| i}, \mathbf{k}_{\| f} \pm \mathbf{q}_{\|}\left|G_{z}\right|^{2} F_{q}^{2}} \\
\hbar \omega_{0}^{*}= & \hbar \omega_{0}\left(\sqrt{q_{\|}^{2}+q_{z}^{2}}\right) \\
& +E_{z i}-E_{z f},
\end{aligned}
$$

where indexes " $\|$ " and " $z$ " are referred to values in the QW plane and perpendicular to it, respectively, $E_{z i}$ and $E_{z f}$ are the subband electron energies in the QW due to quantization of electron momentum $p_{z}=\hbar k_{z}$. In a case of the intrasubband electron-phonon scattering, $E_{z f}=E_{z i}$ and $\hbar \omega_{0}^{*}=\hbar \omega_{0}$. The intersubband electron-phonon interaction was considered in the papers $[2,3]$. The oscillating dependence of the scattering rate due to the change of the intersubband energy on the QW width and the resonance of electron-phonon scattering at $\hbar \omega_{0}=E_{z f}-E_{z i}$ was found [2].

The overlap integral is

$$
\left|G_{z}\right|^{2}=\left|\int \varphi_{e z f} \varphi_{q z} \varphi_{e z i} \mathrm{~d} z\right|^{2}
$$


Here $\varphi_{e z i}$ and $\varphi_{e z f}$ are the $z$ components of the initial and final normalized electron wave functions, respectively. In a single QW of width $L$ with completely reflecting walls (barriers), the $z$ components of phonon wave functions are the standing waves $[6,11-13]$ :

$$
\begin{aligned}
\varphi_{q z}= & \sqrt{\frac{2}{L}} \sin n \frac{\pi}{L} z \text { at } 0<z<L, \\
& n=1,2,3, \ldots, \\
\varphi_{q z}= & 0 \text { at } z>L, z<0 .
\end{aligned}
$$

The square of the normalization coefficient $F_{q}$ of the confined longitudinal PO phonon wave function

$$
\varphi_{q}=F_{q} \varphi_{q z} \mathrm{e}^{\mathrm{i} \mathbf{q}_{\|} \mathbf{r}_{\|}},
$$

as derived from the detailed calculation of the macroscopic confined longitudinal PO phonon potential produced by microscopic $\mathrm{PO}$ atomic vibrations $[11,12]$ is equal to

$$
\begin{gathered}
F_{q n}^{2}=\frac{\hbar \omega_{0}}{A}\left(\frac{1}{\varepsilon_{\infty}}-\frac{1}{\varepsilon_{\mathrm{S}}}\right)\left(q_{\|}^{2}+q_{z n}^{2}\right)^{-1}, \\
q_{z n}=n \frac{\pi}{L}, \quad n=1,2,3, \ldots,
\end{gathered}
$$

where $A$ is the in-plane normalization area.

Further we will take into account only the minimal mode of $q_{z}$ at $n=1$.

The quantization of confined phonon momentum perpendicular to the plane of the QW leads to a great decrease of $F_{q}^{2}$ and, consequently, to the decrease of the electron-phonon scattering rate in the narrow QW. At low QW width, the wave vector $q_{z}=\pi / L$ can exceed the phonon wave vector $q_{\|}$that is obtained from electrons during the electron-phonon interaction: $q_{z}^{2}>q_{\|}^{2}=\left|\mathbf{k}_{\| i}-\mathbf{k}_{\| f}\right|^{2}$. The wave vector $q_{z}=\pi / L$ arises due to confined phonon localization in the QW. It is worth to note that, according to the Heisenberg uncertainty relation,

$$
\Delta p_{z} \Delta z \geq \frac{\hbar}{2}
$$

the phonon momentum cannot be less than $\Delta p_{z \min }=$ $\hbar /(2 \Delta z)$. In the considered case, $\varphi_{q z} \sim \sin n \pi / L z$, $\Delta z=L$, and $q_{z \min }=\pi / L$.

Integration of Eq. (10) over $k_{\| f}$ gives

$$
\begin{aligned}
W_{k_{\| i}}^{ \pm}(\mathrm{QW})= & \frac{W_{0}^{ \pm}}{2 \pi} \int_{0}^{2 \pi} \mathrm{d} \theta\left|G_{z}\right|^{2} \\
& \times\left(k_{f 0}^{2}+k_{\| i}^{2}-2 k_{f 0} k_{\| i} \cos \theta+q_{z}^{2}\right)^{-1},
\end{aligned}
$$

where $\theta$ is the angle between $\mathbf{k}_{\| i}$ and $\mathbf{k}_{f 0}\left(\mathbf{k}_{f 0}\right.$ is defined by Eq. (7)). After integration over $\theta$, we obtain the intrasubband scattering rate of a confined electron with the initial momentum $\hbar \mathbf{k}_{\| f}$ by the ground mode $(n=1)$ of confined phonon in the QW of width $L$ :

$$
W_{k_{\| i}}^{ \pm}(\mathrm{QW})=\frac{W_{0}^{ \pm}\left|G_{z}\right|^{2}}{\sqrt{4 k_{\| i}^{2} q_{i}^{2}+\left( \pm k_{\mathrm{opt}}^{2}-q_{z}^{2}\right)^{2}}} .
$$

Here $q_{z}=\pi / L$, signs "+" and "-" are for the phonon emission and absorption, respectively.

The scattering rate of confined electrons by bulk phonons $\left(q_{z}=0\right)$ is

$$
W_{k_{\| i}}^{ \pm}(\mathrm{QW}, \text { bulk })=\frac{W_{0}^{ \pm}\left|G_{z}\left(q_{z}=0\right)\right|^{2}}{k_{\mathrm{opt}}^{2}} .
$$

We suppose that the saturated electron drift velocity is determined by the scattering rate of electrons with the kinetic energy equal to the phonon energy larger than the PO phonon energy, $E_{c}>\hbar \omega_{0}$ (see Eq. (2)).

At $E_{c} \geq \hbar \omega_{0}$, the scattering rate with phonon emission exceeds the scattering rate with phonon absorption, and the total scattering rates, according to Eqs. (19) and (20), are

$$
\begin{gathered}
W_{\hbar \omega_{0}}^{+}(\mathrm{QW}) \approx \frac{W_{0}^{+}\left|G_{z}\right|^{2}}{k_{\mathrm{opt}}^{2}+q_{z}^{2}}, \\
W_{\hbar \omega_{0}}^{+}(\mathrm{QW}, \text { bulk }) \approx \frac{W_{0}^{+}\left|G_{z}\left(q_{z}=0\right)\right|^{2}}{k_{\mathrm{opt}}^{2}} .
\end{gathered}
$$

Due to a weak dependence of $W_{k}^{+}$on the electron energy, we suppose $W_{k}^{+}\left(E>\hbar \omega_{0}\right) \approx W_{k}^{+}\left(E \approx \hbar \omega_{0}\right)$. Consequently, the ratio of the velocity of electrons (with the energy $E_{e}=\hbar \omega_{0}$ ) confined in the QW and scattered by confined phonons, to that scattered by bulk phonons can be estimated as

$$
\begin{aligned}
\frac{v_{\mathrm{sat}}(\mathrm{QW})}{v_{\mathrm{sat}}(\mathrm{QW}, \mathrm{bulk})} & \approx \frac{W_{\hbar \omega_{0}}(\mathrm{QW}, \text { bulk })}{W_{\hbar \omega_{0}}(\mathrm{QW})} \\
& =\frac{\left|G_{z}\left(q_{z}=0\right)\right|^{2}}{\left|G_{z}\right|^{2}}\left(1+\frac{q_{z}^{2}}{k_{\mathrm{opt}}^{2}}\right) .
\end{aligned}
$$

At $q_{z}=\pi / L_{q}$, where $L_{q}$ is the phonon QW width, and $k_{\mathrm{opt}}=\pi / L_{\mathrm{opt}}$,

$$
\frac{\left|q_{z}\right|^{2}}{k_{\mathrm{opt}}^{2}}=\left(\frac{L_{\mathrm{opt}}}{L_{q}}\right)^{2} .
$$

For $\Gamma$ valley electrons in GaAs, $L_{\mathrm{opt}}=\pi / k_{\mathrm{opt}}=$ $12.6 \mathrm{~nm}$. A tenfold increase of the maximal drift velocity in the QW can be obtained at $L_{q}=4 \mathrm{~nm}$. 
One can see that even with the contribution of the overlap integral ignored the phonon quantization gives a great increase of the maximal drift velocity.

The overlap integrals $\left|G_{z}\right|^{2}$ and $\left|G_{z}\left(q_{z}=0\right)\right|^{2}$ increase with decreasing the QW width [11]. This increase has to be taken into account in Eq. (22). However, at $L<L_{\mathrm{opt}}$ this increase of $\left|G_{z}\right|^{2}$ is negligible in comparison with the decrease of $W_{k}(\mathrm{QW})$ due to the increase of $z$ component of the phonon momentum.

\section{Phonon confinement and the Heisenberg uncertainty}

Above, we have considered the case when the $z$ component of the phonon wave function is a standing wave (Eq. (14)). In this case, the localization area of the phonon wave function is defined as $\Delta z=L$, and the minimal value of the phonon momentum is determined according to Heisenberg uncertainty $\Delta p_{z} \Delta z \geq h / 2$ as $q_{z} \geq \pi / L$.

For the evaluation of $\Delta p_{z}$ in a general case, let us employ the more precise Heisenberg uncertainty equations with the inaccuracies as mean square deviations from the average values of momentum $\bar{p}$ and coordinate $\bar{z}[15]$ :

$$
\begin{aligned}
& \overline{\left(\Delta p_{z}\right)^{2}}=\int\left(p_{z}-\overline{p_{z}}\right)^{2}\left|\psi\left(p_{z}\right)\right|^{2} \mathrm{~d} p_{z}, \\
& \overline{(\Delta z)^{2}}=\int(z-\bar{z})^{2}|\varphi(z)|^{2} \mathrm{~d} z,
\end{aligned}
$$

where

$$
\overline{p_{z}}=\int p_{z}\left|\psi\left(p_{z}\right)\right|^{2} \mathrm{~d} p_{z}, \quad \bar{z}=\int z|\varphi(z)|^{2} \mathrm{~d} z,
$$

and the wave functions in the space of momentum $\psi\left(p_{z}\right)$ and coordinate $\varphi(z)$ are constrained by the Fourier transforms:

$$
\begin{aligned}
\psi\left(p_{z}\right) & =\frac{1}{\sqrt{2 \pi \hbar}} \int \varphi(z) \exp \left(\frac{-\mathrm{i} p_{z} z}{\hbar}\right) \mathrm{d} z, \\
\varphi(z) & =\frac{1}{\sqrt{2 \pi \hbar}} \int \psi\left(p_{z}\right) \exp \left(\frac{\mathrm{i} p_{z} z}{\hbar}\right) \mathrm{d} p_{z} .
\end{aligned}
$$

From Eqs. (23)-(27) the Heisenberg uncertainty can be defined as

$$
\overline{\left(\Delta p_{z}\right)^{2}} \cdot \overline{(\Delta z)^{2}} \geq \frac{\hbar^{2}}{4} \quad \text { or } \quad \Delta p_{z}^{*} \cdot \Delta z^{*} \geq \frac{\hbar}{2} .
$$

Here $\Delta z^{*}=\sqrt{\overline{(\Delta z)^{2}}}$ and $\Delta p_{z}^{*}=\sqrt{\overline{\left(\Delta p_{z}\right)^{2}}}$.
For a minimal $\Delta p_{z}^{*}$ value we obtain

$$
\Delta p_{z}^{*}=\frac{\hbar}{2 \sqrt{\overline{(\Delta z)^{2}}}},
$$

where $\overline{(\Delta z)^{2}}$ is defined by Eq. (24).

Namely, this value of $\Delta p_{z}^{*}=\hbar q_{z}$ determines the decrease of the factor $F_{q}$ (Eq. (16)) and consequently, the great increase of the maximal drift velocity (Eq. (22)) in a narrow phonon $\mathrm{QW}$.

For a phonon standing wave $\varphi_{q}(z)$, Eq. (24) gives $\overline{(\Delta z)^{2}}=L^{2}$ or $q_{z}=\pi / L$. Note that in spite of $\overline{p_{z}}=0$, fluctuation of the phonon momentum $\Delta p_{z}$ is an additional source of drift velocity fluctuations.

\section{Electron-interface PO phonon scattering}

Heterointerfaces of double heterostructures give rise to the confinement of optical phonons located in the middle of a QW as well as interface (IF) modes, which are located in the vicinity of the interfaces $[11,13]$. The total electron-phonon scattering rate in the QW is a sum of the scattering rates by confined and IF phonons:

$$
W_{\text {total }}=W_{k}(\mathrm{QW})+W_{k}(\mathrm{IF}) .
$$

In double heterostructures, the sum rule for the scattering rate form factors holds [11]:

$$
\left|G_{z}\left(q_{z}=0\right)\right|^{2}=\left|G_{z}(\mathrm{QW})\right|^{2}+\left|G_{z}(\mathrm{IF})\right|^{2} .
$$

The sum rule means that if we neglect the difference in eiqenfrequencies of phonon modes, due to the existence of the interface, namely, if we neglect the difference in coupling constants of phonon modes, the total confined electron scattering rate by confined and interface phonons is the same as that for scattering rate by bulk phonons,

$$
W_{k}(\mathrm{QW}, \text { bulk }) \approx W_{k}(\mathrm{QW})+W_{k}(\mathrm{IF}) \equiv W_{\text {total }} .
$$

Consequently, a great decrease of confined electronconfined phonon scattering rate with decreasing QW width $L$ is exactly compensated by the increase of confined electron-IF phonon scattering rate. It takes place, for example, in AlAs / GaAs / AlAs heterostructure due to a small difference in the coupling constants of phonon modes of AlAs and GaAs [11].

As a result, the increase of the maximal drift velocity due to the decrease of $W_{k}(\mathrm{QW})$ in a narrow $\mathrm{QW}$ disappears due to the increase of scattering rate by IF phonons.

The compensation effect can be decreased by choosing a semiconductor material of the heterostructure 


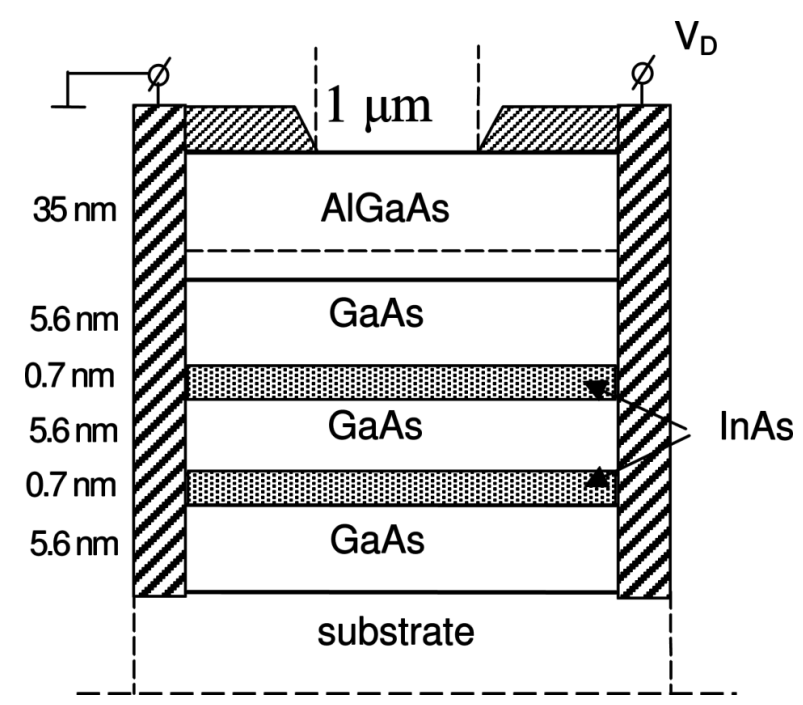

Fig. 1. Sequence of layers for AlGaAs / GaAs heterostructures with incorporated InAs layers having QDs. The dotted line shows a Si $\delta$-doped layer $\left(2 \cdot 10^{12} \mathrm{~cm}^{-2}\right)$.

with a larger difference in electron-phonon coupling constants, as it is, for example, in a case of InAs/GaAs/InAs and AlSb/InAs / AlSb. If the twodimensional electron gas is formed in a homopolarpolar-homopolar heterostructure, Ge/InAs/Ge, the total electron-phonon scattering rate is approximately equal to the scattering rate by confined phonons $W_{k}(\mathrm{QW})$, and the influence of IF scattering is negligible [11].

With the aim to increase the saturated electron velocity we propose to develop an InAs / GaAs / InAs structure with thin (two monolayers) InAs barrier layers with a high density of quantum dots (QDs) inserted in the GaAs QW. Moreover, we assume that chaotic destruction of the GaAs / InAs interface by QDs eliminates inelastic electron scattering by IF PO phonons.

\section{The giant increase of electron drift velocity (experiment)}

Figure 1 demonstrates schematically the modulation doped AlGaAs / GaAs / InAs / GaAs / InAs / GaAs heterostructures grown by molecular beam epitaxy on a GaAs semi-insulating substrate. In a thin InAs pseudomorphic layer, the self-assembled InAs quantum dots were formed. The QD size is around $5 \times 40 \mathrm{~nm}^{2}$ [16]. The width of the InAs / GaAs / InAs PO phonon QW of 3-5 nm corresponds, according to Eq. (22), to the tenfold increase of the saturated drift velocity. Two types of the structures were grown: S1 with high concentration of QDs of $3 \cdot 10^{10} \mathrm{~cm}^{-2}$ and S2 with low concentration of QDs of $10^{10} \mathrm{~cm}^{-2}$ [4].

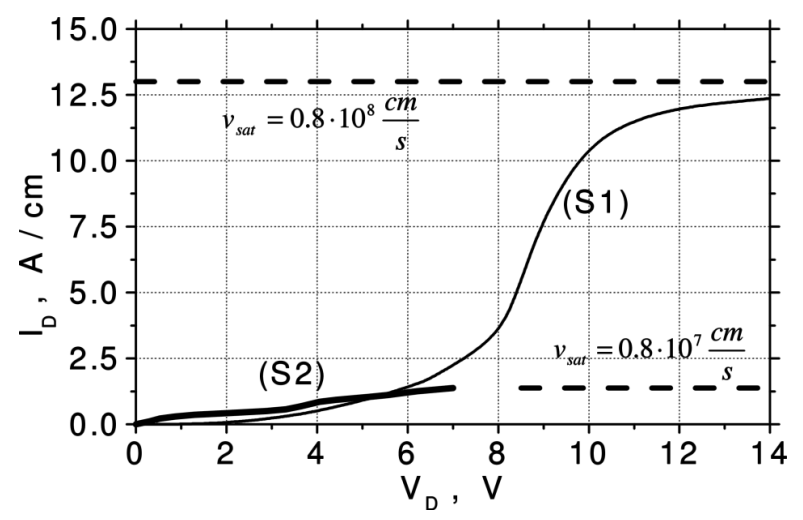

Fig. 2. Current-voltage characteristics of AlGaAs/GaAs heterostructures with incorporated InAs layers with high (S1) and low (S2) concentration of InAs QDs.

Figure 2 shows the measured $I-V$ dependences of two types of structures [14]. At low voltages, before ionization of QDs, the current through structure S1 is much less than that through structure S2 because of a lower free electron concentration. At higher voltages, the increase of the current, due to ionization of QDs at high electric field, in structure S1 exceeds that increase in structure $\mathrm{S} 2$. The current in structure S2 is saturated at the level with the drift velocity of less than $10^{7} \mathrm{~cm} / \mathrm{s}$. The current in structure $\mathrm{S} 1$ is saturated at $13 \mathrm{~A} / \mathrm{cm}$ or at $n_{S} v_{\text {sat }}=0.8 \cdot 10^{20} \mathrm{~cm}^{-1} \mathrm{~s}^{-1}$.

Since the sheet electron concentration is not larger than $10^{12} \mathrm{~cm}^{-2}$, we have to assume that the saturated drift velocity is $0.8 \cdot 10^{8} \mathrm{~cm} / \mathrm{s}$. This is ten times larger as the saturated velocity $0.8 \cdot 10^{7} \mathrm{~cm} / \mathrm{s}$ in bulk GaAs at an electric field higher than $60 \mathrm{kV} / \mathrm{cm}$.

We assume that such a giant increase of the saturated drift velocity is due to both the confinement of PO phonons in AlGaAs / GaAs / InAs / GaAs / InAs QWs and the suppression of the GaAs / InAs IF phonon mode by forming a large number of InAs QDs.

\section{Conclusions}

1. The drift velocity increase in high electric fields in common semiconductors is limited by inelastic optical phonon scattering: an electron loses the drift velocity acquired in an electric field by optical phonon emission. Therefore, the maximal possible drift velocity in semiconductors can be evaluated as $v_{\text {sat }} \approx$ $\sqrt{\hbar \omega_{0} / m}$.

2. Dimensional phonon and electron confinement increases the electron saturated drift velocity in the QW: 


$$
\begin{aligned}
v_{\text {sat }}(\mathrm{QW})= & \frac{\left|G_{z}\left(q_{z}=0\right)\right|^{2}}{\left|G_{z}\right|^{2}}\left(1+\frac{\left(\Delta p_{z}^{*}\right)^{2}}{2 m \hbar \omega_{0}}\right) \\
& \times v_{\text {sat }}(\mathrm{QW}, \text { bulk }),
\end{aligned}
$$

where $\Delta p_{z}^{*}$ is the minimal quantized phonon momentum perpendicular to the QW plane, which is determined by localization of the PO phonon in the real space $\Delta z^{*}$. According to the Heisenberg uncertainty, $\Delta p_{z}^{*}=\hbar /\left(2 \Delta z^{*}\right)$, where $\Delta z^{*}=\sqrt{\overline{(\Delta z)^{2}}}$. Namely, the great increase of $\Delta p_{z}^{*}$ is responsible for the great increase of the saturated drift velocity in a narrow QW.

3. The experimental observation of a tenfold increase of $v_{\mathrm{sat}}$ in the AlGaAs/GaAs/InAs/GaAs/InAs heterostructure is in agreement with the theoretical prediction.

\section{References}

[1] J. Požela, V. Jucienè, and K. Požela, Confined electron-optical phonon scattering rates in $2 \mathrm{D}$ structures containing electron and phonon walls, Semicond. Sci. Technol. 10, 1076-1083 (1995).

[2] J. Požela, K. Požela, and V. Jucienè, Electron mobility and electron scattering by polar optical phonons in heterostructure quantum wells, Semiconductors 34, 1011-1015 (2000).

[3] J. Požela, A. Namajūnas, K. Požela, and V. Jucienė, Polar optical phonon confinement and electron mobility in quantum wells, Physica E 5, 108-116 (1999).

[4] J.K. Požela and V.G. Mokerov, Giant increase of electron maximum drift velocity in a channel of heterostructure field-effect transistor, Fiz. Tekh. Poluprovodn. 39, 362-366 (2006).

[5] V.L. Bonch-Bruevich and S.G. Kalashnikov, Semiconductor Physics (Nauka, Moscow, 1977) p. 532, Eqs. 6.4-6.6 [in Russian].

[6] P. Yu and M. Cardona, Fundamentals of Semiconductors (Fiziko-matematicheskaya Literatura, Moscow, 2002) p. 209, Eqs. 5.67-5.69 [in Russian].
[7] A. Matulionis, J. Požela, and A. Reklaitis, Dynamics of electron heating, in: Electrons in Semiconductors, Vol. 1, Many-Valley Semiconductors (Mokslas, Vilnius, 1978) pp. 7-58 [in Russian].

[8] A. Matulionis, J. Požela, and A. Reklaitis, Drift velocity oscillations in $n$-GaAs at $77 \mathrm{~K}$, Phys. Status Solidi A 31, 83-87 (1975).

[9] A. Matulionis, J. Požela, and A. Reklaitis, Monte Carlo calculations of hot-electron transient behaviour in CdTe and GaAs, Phys. Status Solidi A 35, 43-48 (1976).

[10] V. Gantmacher and I.B. Levinson, Scattering of Charge Carriers of Metals and Semiconductors (Nauka, Moscow, 1984) [in Russian].

[11] N. Mori and T. Ando, Electron-optical phonon interaction in single and double heterostructures, Phys. Rev. B 40, 6175-6188 (1989).

[12] I. Lee, S.M. Goodnick, M. Gulia, E. Molinari, and P. Lugli, Microscopic calculation of the electron-optical phonon interaction in ultrathin $\mathrm{GaAs} / \mathrm{Al}_{x} \mathrm{Ga}_{1-x}$ As alloy quantum-well systems, Phys. Rev. B 51, 7046-7057 (1995).

[13] C.R. Bennett, M.A. Amato, N.A. Zakhleniuk, B.K. Ridley, and M. Babiker, Effects of monolayer on the electron-phonon scattering rates in a quantum well: Dielectric continuum versus hybrid model, J. Appl. Phys. 83, 1499-1506 (1998).

[14] V.G. Mokerov, J. Pozela, K. Pozela, and V. Juciene, Giant increase of electron saturated drift velocity in a MODFET channel, in: Proceedings of 14th International Conference on Nonequilibrium Carrier Dynamics in Semiconductors (HCIS-14) (Chicago, USA, July 24-29, 2005, in press).

[15] O.I. Zav'yalov, Sootnoshenie neopredelennostey, in: Fizicheskii Enciklopedicheskii Slovar' (Sovetskaya Enciklopediya, Moscow, 1965) p. 580 [Uncertainty relation, in: Physical Encyclopaedical Dictionary, in Russian].

[16] V.G. Mokerov, Yu.V. Fedorov, L.E. Velikovski, and M.Yu. Scherbakova, New quantum dot transistor, Nanotechnology 12, 552-555 (2001). 


\title{
ELEKTRONU SOTIES DREIFINIO GREIČIO PADIDĖJIMAS KVANTINĖJE DUOBĖJE DE்L POLINIŲ OPTINIŲ FONONŲ SUSPRAUDIMO
}

\author{
J. Požela, K. Požela, V. Jucienė
}

Puslaidininkiu fizikos institutas, Vilnius, Lietuva

\section{Santrauka}

Parodyta, kad suspraustu polinių optinių fononų impulso kvantavimas sąlygoja žymų elektronų soties dreifo greičio kvantineje duobeje padidejimą, palyginus su tuo greičiu tūriniame puslaidininkyje.

Iprastuose puslaidininkiuose elektronų dreifo greičio didèjimą stipriuose elektriniuose laukuose apriboja netampri elektronu sklaida optiniais fononais: igytą elektriniame lauke dreifo greiti elektronas praranda emituodamas optini fononą. Todèl maksimaliai galimas dreifo greitis gali būti aprašytas taip: $v_{\text {sat }} \approx \sqrt{\hbar \omega_{0} / m}$. Fononu suspraudimas kvantinejje duobejje padidina elektronų soties dreifini greiti kvantinèje duobejje:

$$
v_{\text {sat }}(\mathrm{QW})=\frac{\left|G_{z}\left(q_{z}=0\right)\right|^{2}}{\left|G_{z}\right|^{2}}\left(1+\frac{\left(\Delta p_{z}^{*}\right)^{2}}{2 m \hbar \omega_{0}}\right) v_{\text {sat }}(\mathrm{QW}, \text { bulk }),
$$

čia $\Delta p_{z}^{*}$ - minimalus kvantuotas fonono impulsas, statmenas kvantinès duobės plokštumai, kuris yra sąlygotas PO fonono lokalizacijos koordinačiu erdveje dydžio $\Delta z^{*}$. Kaip seka iš Heizenbergo nelygybès, $\Delta p_{z}^{*}=\hbar /\left(2 \Delta z^{*}\right)$, kur $\Delta z^{*}=\sqrt{\overline{(\Delta z)^{2}}}$.

Būtent $\Delta p_{z}^{*}$ didèjimas siauroje kvantinèje duobejje yra atsakingas už dideli elektrono soties dreifo greičio augimą.

Taigi, yra paaiškintas elektronu dreifo greičio soties reikšmès padidejimas dešimteriopai, stebimas eksperimentiškai $\mathrm{AlGaAs} / \mathrm{GaAs} / \mathrm{InAs} / \mathrm{GaAs} / \mathrm{InAs}$ ivvairialyčiame darinyje. 